\title{
Incorporating Delayed Neutrons Into The Point-Model Equations Routinely Used For Neutron Coincidence Counting In Nuclear Safeguards
}

\author{
S. Croft ${ }^{\mathrm{a}}$, A. Favalli ${ }^{\mathrm{b}}$ \\ ${ }^{a}$ Safeguards \& Security Technology \\ Nuclear Security and Isotope Technology Division \\ One Bethel Valley Road \\ PO Box 2008, MS-6166, Oak Ridge, TN 37831-6166 USA \\ ${ }^{\mathrm{b}}$ Safeguards Science \& Technology Group \\ Non-proliferation and Nuclear Engineering Division \\ Los Alamos National Laboratory \\ MS E540, Los Alamos, NM 87545 USA
}

\begin{abstract}
We extend the familiar Bö hnel point-model equations, which are routinely used to interpret neutron coincidence counting rates, by including the contribution of delayed neutrons. After developing the necessary equations we use them to show, by providing some numerical results, what the quantitative impact of neglecting delayed neutrons is across the full range of practical nuclear safeguards applications. The influence of delayed neutrons is predicted to be small for the types of deeply sub-critical assay problems which concern the nuclear safeguards community, smaller than uncertainties arising from other factors. This is most clearly demonstrated by considering the change in the effective $(\alpha, n)$-to-spontaneous fission prompt-neutron ratio that the inclusion of delayed neutrons gives rise to. That the influence of delayed neutrons is small is fortunate, and our results justify the long standing practice of simply neglecting them in the analysis of field measurements.
\end{abstract}

Keywords: delayed neutrons; neutron coincidence counting; point-model equations; neutron multiplicity counting. 


\section{Introduction}

32 Neutron coincidence counting (NCC) is a well-established and widely used technique for non33 destructively assaying the plutonium content of items [1-3]. The conventional target quantity of the assay 34 is the ${ }^{240} \mathrm{Pu}$-effective mass, a weighted linear sum of ${ }^{238} \mathrm{Pu},{ }^{240} \mathrm{Pu}$ and ${ }^{242} \mathrm{Pu}$. The total plutonium mass can 35 then be derived from the ${ }^{240} \mathrm{Pu}$-effective mass from independent knowledge of the isotopic composition of the plutonium. The primary measured quantities in NCC are the singles and doubles rates. The singles count rate, $S$, is the total neutron time-averaged event rate on the pulse train and is often referred to as the totals rate. The doubles (reals, pairs or coincidence) rate, $D$, is most often determined using a form of autocorrelation analysis known as shift-register logic [1]. For practical non-destructive assay applications the two rates are expressed in terms of a combination of item and detector parameters, and nuclear data constants, using algebraic expressions based on a one-group, point, prompt-kinetics model [4-11] - the so called Bö hnel point-model equations [1-4]. These can then be solved for the ${ }^{240} \mathrm{Pu}$-effective mass. The standard formulation of the point-model equations neglects delayed neutrons, this is an approximation. In this article we quantify what impact this routinely applied approximation has on assay results across the domain of nuclear safeguards.

Our starting point is the familiar point-model equations for the singles and doubles counting rates. These may be expressed as follows:

where the variables used are defined as follows:

$F_{S}=$ the effective ${ }^{240} \mathrm{Pu}$ spontaneous fission rate $=m_{\text {eff }} \cdot g$, the product of the ${ }^{240} \mathrm{Pu}$-effective mass,

$55 m_{e f f}$, and $g$, the specific spontaneous fission rate of ${ }^{240} \mathrm{Pu}$

$56 \varepsilon=$ the neutron detection efficiency (probability of detection per neutron emerging from the item)

$57 v_{S i}=i^{\text {th }}$ factorial moment of the spontaneous fission neutron distribution

$58 v_{I i}=i^{\text {th }}$ factorial moment of the induced fission neutron distribution

$59 M_{L}$ is the neutron leakage self-multiplication $M_{L}=M_{T} \cdot p_{L}=\left(1-p_{f}-p_{c}\right) /\left(1-v_{I 1} p_{f}\right)$ where $M_{T}=1 /\left(1-v_{I 1} p_{f}\right)$ is the total self-multiplication, $p_{L}=1-p_{f}-p_{c}$ is the probability of neutron leakage,

61 where $p_{f}$ and $p_{c}$ are the probabilities for a neutron to undergo fission and parasitic captures [10,11,12].

$62 \alpha=$ the $(\alpha, n)$-to-spontaneous fission prompt-neutron production ratio,i.e. $(\alpha, n)$-rate $/\left(F_{S} \cdot v_{S I}\right)$

$63 f_{d}=$ the doubles gate utilization factor $[2,8,13]$

64 
For the present discussion we imagine the common application in which the properties of the detector (efficiency and gate utilization factor) are assumed to be known from calibration and the $\alpha$-value can be calculated from the isotopic composition of the $\alpha$-emitting constituents of the measurement items (e.g. ${ }^{238} \mathrm{Pu},{ }^{239} \mathrm{Pu},{ }^{240} \mathrm{Pu},{ }^{241} \mathrm{Pu},{ }^{242} \mathrm{Pu}$, and ${ }^{241} \mathrm{Am}$ ) and chemical form (e.g. dioxide) of the chemically pure compound inside the item.

\section{Derivation of Böhnel -like point model equation with delayed neutrons embedded}

\subsection{Delayed neutrons and assumptions in the model}

Passive neutron coincidence counting assays are performed in steady state conditions when the neutron emission processes have settled into a quiescent dynamic equilibrium. Thus we can speak in terms of the time averaged behavior. Delayed neutrons are emitted long after the associated prompt fission neutrons (which for our discussion are emitted essentially instantaneously at the time of fission), on a typical time scale of $\sim 1 / 10-100 \mathrm{~s}[14,15]$. This is also much longer than the time prompt fission neutrons take to emerge from measurement item and subsequently persist in the detector system (for commonly used moderated ${ }^{3} \mathrm{He}$ based detection systems the characteristic detector die-away time is of the order of 10's $\mu \mathrm{s})[1,7,11]$. Thus, as a practical matter, the fundamental assumption we introduce in our treatment is that DN's have the essential characteristics of time random neutrons, like $(\alpha, n)$ neutrons, being created one at a time and independent in time from the parent fission events (see also [22,23] for discussion of delayed neutrons in a reactor close to critical) This is a key assumption in order to introduce DN contributions into the Bö hnel-like point model equations, as we will do in the next chapter. For the discussion, it is also worth noting that, in the Böhnel point-model equations, time never appears explicitly. One derives first the distribution of the total number of neutrons leaving the sample per one starting neutron, and after that per one source event (spontaneous fission neutrons with a number distribution), assuming that all these neutrons (including internal multiplication before leakage) are all generated "instantaneously" (the idea of "superfission" introduced by Böhnel [4]). Time, in the form of count rates of singles, doubles etc., which means detection per unit time, enters only by assuming a source intensity for the spontaneous fissions (and of course $(\alpha, n)$ neutrons), but assuming that the fission chains do not overlap (the concept of superfission guarantees this, since the evolution of the chains takes zero time). Hence the doublets, triplets etc. are calculated from the generation rate of the multiplets and the detection probability of neutrons. Finite (between zero and unity) gate factors account for the use of finite coincidence gates and the finite residence time of neutrons in the detector. Delayed neutrons, however, by definition, cannot be accommodated into the concept of superfission. But our assumption that all neutrons which originate from a delayed neutron precursor represent only time-uncorrelated 'background' allows us to introduce the DN contribution in the Böhnel point-model equations like a special kind of $(\alpha, n)$ contribution. By this assumption we avoid having to deal explicitly with connected time-dependent processes, because neutrons belonging to the prompt-fission chain are separated from those generated by delayed neutron precursors. The former gives the correlated counts, the latter the corresponding uncorrelated background. A full derivation by master neutron stochastic equation is in preparation [24], but it goes beyond the scope of this paper, which is ultimately to quantify the contribution of the delayed neutron production in the Böhnel point-model equations and applied in the nuclear safeguards and also waste management assay scenarios. 
Each spontaneous fission (SF) gives rise to an average of $v_{\mathrm{Sd}} \mathrm{DN}$ 's, and each induced fission (IF) gives rise to an average of $v_{\mathrm{Id}}$ DN's. In the one neutron energy-group point-model [11], each neutron initially released inside the measurement item also results, again on average, in a number of IF's given by:

where:

$p_{f}$ is the probability that the initial neutron history will end in absorption resulting in fission; $M_{T}$ is the total self-multiplication $=1 /\left(1-v_{I 1} p_{f}\right)$ and, the approximate form on the right hand side holds when the probability of parasitic $p_{\mathrm{c}}$ (e.g. (n, $\left.\mathrm{Y}\right)$ ) neutron capture in the item relative to the corresponding probability of induced fission $p_{f}$ is small.

In nuclear safeguards of plutonium, the items of interest are usually deeply sub-critical [1,2], and, we are concerned with quantifying the mass of $\mathrm{Pu}$ present through the ${ }^{240} \mathrm{Pu}$-effective mass which is used as a measure of the overall SF rate. We shall therefore treat the SF process as the primary initiating event and scale primary ('source term') neutron production from it. Now, on the average $F_{S}$ SF's take place per second and these in turn liberate $\left(\mathrm{F}_{\mathrm{S}} v_{\mathrm{S} 1}\right)$ prompt neutrons per second in to the system along with $\left(\mathrm{F}_{\mathrm{S}} v_{\mathrm{Sd}}\right)$ delayed neutrons, and $\left[\left(\mathrm{F}_{\mathrm{S}} v_{\mathrm{S} 1}\right) \alpha\right](\alpha, n)$ neutrons. Thus, the rate at which primary-source neutrons are released into the measurement item is given by:

$$
F_{S} v_{S 1}\left(1+\alpha+\frac{v_{S d}}{v_{S 1}}\right)
$$

and hence the rate of IF DN production is obtained by the product of Eq.(4), Eq.(3), and the IF DN yield as follows:

$F_{S} v_{S 1}\left(1+\alpha+\frac{v_{S d}}{v_{S 1}}\right)\left(\frac{M_{L}-1}{v_{I 1}-1}\right) v_{I d}$

The IF DN contribution given by Eq.(5), like the SF DN's, have the essential (mathematical) characteristics of additional $(\alpha, n)$ events. Thus, in the standard point-model equations, Eq.(1) and Eq.(2), we should replace the term $(1+\alpha)$ by the following term:

$$
\left(1+\alpha+\frac{v_{S d}}{v_{S 1}}\right)\left(1+\left(\frac{M_{L}-1}{v_{I 1}-1}\right) v_{I d}\right)
$$

For non-multiplying items the second factor in brackets reduces to zero. For chemically pure metallic items we would expect $(\alpha, n)$ production to be close to zero and so, the time-random neutron production will be influenced most strongly by the DN component for such materials. This may be the case for certain calibration items, for example. 
Including DN production gives rise to revised expressions for the $\mathrm{S}$ and $\mathrm{D}$ rates that are more complicated than the traditional forms when one wishes to solve for $\mathrm{M}_{\mathrm{L}}$. But, for weakly multiplying items ( $\left(\mathrm{M}_{\mathrm{L}}\right.$ $1)<1$, which is typical of safeguards and waste measurements) because both $v_{\mathrm{Sd}} / v_{\mathrm{S} 1}$ and $v_{S d} /\left(v_{I 1}-1\right)$ are small, we can solve them iteratively, incrementing the value of $(1+\alpha)$ to take account of the DN contribution.

The question is whether the revised estimate for the time-random neutron production contribution makes a substantive difference to the overall quality of the mass assay, which is, after all, the objective of the measurement. To examine this question we make some numerical estimates. We shall consider the fractional change in $(1+\alpha)$ due to the inclusion of DN's, namely the quantity:

Eq.(7) is simply Eq.(6) divided by the nominal or conventional value of $(1+\alpha)$. The extreme case is immediately evident as $\alpha=0$ in the limit $M_{L} \gg 1$. This is unrealistic from a safeguards perspective but 168 provides a clear upper bound.

169 For the SF of ${ }^{240} \mathrm{Pu}$ we take $v_{\mathrm{Sd}} / v_{\mathrm{S} 1} \cong(0.00675 / 2.154)=0.00313$, and for IF we adopt ${ }^{239} \mathrm{Pu}$ with $v_{\mathrm{Id}} /\left(v_{\mathrm{II}}{ }^{-}\right.$ $1) \cong(0.00645 /(3.009-1)=0.00321$. The average prompt neutron choices are based on the prompt neutron multiplicity distribution evaluation work of Holden and Zucker [16] in the case of ${ }^{240} \mathrm{Pu}$, and in the case of ${ }^{239} \mathrm{Pu}$ we have assumed induced fission by incoming 1-MeV neutrons and used the tables of Zucker and Holden [17]. The delayed neutron yield for ${ }^{240} \mathrm{Pu}$ was estimated using the 'systematics' relation of Tuttle [13]. Where data exists this relation is believed to be generally accurate to about $15 \%$. For ${ }^{239} \mathrm{Pu}$ the DN yield for fast neutron induced fission as reported by England et [19] (the ENDF/B-V value) was selected because it is still in common use (e.g. in the Monte Carlo code MCNPX [20]).

\begin{tabular}{|r|r|c|}
\hline \multicolumn{1}{|l|}{$\alpha$} & \multicolumn{1}{|c|}{$\mathrm{L}_{\mathrm{L}}$} & Fractional Change in $(1+\alpha)$ \\
\hline 0 & 1 & 1.0031 \\
\hline 0 & 1.05 & 1.0033 \\
\hline 0 & 1.1 & 1.0035 \\
\hline 0 & 1.2 & 1.0038 \\
\hline 0 & 1.3 & 1.0041 \\
\hline 0 & 2 & 1.0064 \\
\hline 0 & 3 & 1.0096 \\
\hline 0 & 4 & 1.0128 \\
\hline 0 & 100 & 1.322 \\
\hline 0.5 & 4 & 1.0117 \\
\hline 1 & 4 & 1.0112 \\
\hline 3 & 4 & 1.0104 \\
\hline 10 & 4 & 1.0099 \\
\hline
\end{tabular}


Table 1. A short table of computed results for the fractional change in the factor $(1+\alpha)$ caused by the inclusion of delayed neutrons as a function of leakage self-multiplication and $\alpha$, for a range of values of general safeguards interest.

From Table 1, generated using Eq.(7), we see that the estimated fractional change in the conventional value of $(1+\alpha)$ which should be used in the so-called known- $\varepsilon$ (and other detector parameters) and known$\alpha$ (i.e. calculated $\alpha$ ) solution of the $S$ and D point-model equations is small for the typical small mass, weakly multiplying $\mathrm{Pu}$ product items of routine safeguards concern. For a given item the uncertainty in the calculation of $(1+\alpha)$ is likely to exceed $1 \%$ even under near ideal conditions, the best case from a nuclear data perspective being pure $\mathrm{PuO}_{2}$. The sensitivity calculation can be undertaken as follows: $\mathrm{y}=(1+\alpha), \mathrm{dy}=\mathrm{d} \alpha$, and $\mathrm{dy} / \mathrm{y}=\mathrm{d} \alpha /(1+\alpha)$, and so $\frac{\sigma_{y}}{y}=\left(\frac{\alpha}{1+\alpha}\right) \frac{\sigma_{\alpha}}{\alpha}$ based on the familiar first order Taylor series expansion approach to the propagation of variance. For pure $\mathrm{PuO}_{2}$ product the value $(1+\alpha)$ is typically in the range 0.5 to 2 , depending on the isotopic composition, and the best $(1+\alpha)$-value estimates are based on $(\alpha, n)$ yield measurements that have a fractional uncertainty of about $1.2 \%$. Uncertainty in the isotopic composition, often based off high-resolution gamma-ray spectrum analysis, introduces additional item-specific uncertainty. We just state these results to provide context and without justification because, in practice, it is our experience, that the presence of unknown levels of unknown light-element impurities can influence the value of $(1+\alpha)$, for a given item, to a greater degree than these contributions. A modest increase of $2 \%$ to $5 \%$ in the value of a might be considered typical, or even normal, and is often handled inherently through the use of calibration items similar to those to be measured. But it is the unknown variation from item-to-item at the percent level which we are comparing the delayed neutron bias against.

Note we have taken $\alpha=0$ (pure metal), $M_{L}=4$ ("high mass") to be a realistic extreme for measurements of routine safeguard interest For context, Krick et al [25], considers Pu masses up to $4 \mathrm{~kg}$ for which $M_{L}$ is less than $\sim 2.5$, and these already represent challenging cases for quantitative NCC. Of course for $\alpha=0$ the fractional change in $(1+\alpha)$ can be driven larger by raising $M_{L}$ to arbitrarily large levels (for example, it exceeds a factor of 10 for $M_{L} \approx 2800$ using the current parameters), but this is not our domain of application. This apparent potential for highly deviant behavior is also just an artifact of the way we have chosen to present the results in terms of the fractional change in conventional $(1+\alpha)$. We did this because it is then easy to see that the change due to the inclusion of delayed neutrons is small compared to other sources of uncertainty in this term. For example, to restate our earlier remark in a different way, in metallic items low atomic number impurities at the ppm level by mass may typically raise $(1+\alpha)$ from the ideal value of unity to a few percent above unity. It is not possible to account for impurities in the known- $\alpha$ calculation and so one can appreciate that a percent uncertainty is well within our ability to compute $(1+\alpha)$ in such cases, which immediately illustrates that the DN contribution is modest and justifies why, the standard neutron coincidence counting expressions, which ignore the DN contribution, may still be applied successfully for routine safeguards measurements (especially when representative standards are available from which effective analysis model calibration-parameters can be extracted). It should none-the-less still be appreciated that even in the $\alpha=0$ and high $M_{L}$ limit, that is when the only neutrons in the system can be tracked back to induced fission events, the overall fraction of 
neutrons due to DN's will continue to remain small. This is because the DN yield relative to the prompt neutron yield is small. They will have different importance because of their softer energy spectrum and for high quality criticality benchmarks DN's certainly need to be treated in appropriate detail as a consequence. Delayed neutrons are also crucial to reactor control. But these considerations are far from the deeply sub-critical measurement problems with which we are concerned with in nuclear safeguards and our chosen analysis methods was sufficient to cover this application.

\section{Conclusion and remarks}

The traditional formalism for interpreting neutron correlation counting data for the passive nondestructive assay of $\mathrm{Pu}$ materials currently relies on the use of point-model equations that do not include delayed neutron production. DN's are emitted long after fission with periods much greater than the characteristic time constants at work in the measurement item and detector and so behave like an additional weak source of time random neutrons. The intensity depends on the leakage self-multiplication, $M_{L}$, of the item and the induced fission contrition scales with the induced fission prompt signal. Having the character of random emissions they may be treated approximately as an additional $(\alpha, n)$ contribution in the conventional point-model equations. In this paper we have taken the step to quantify the impact by suitably modifying the $(1+\alpha)$ factor. At the time of solution (inversion) of the modified point-model equations, that is when performing an assay for ${ }^{240} \mathrm{Pu}$-effective mass, the change is easy to implement iteratively, although the influence is expected to always be small. For low multiplication safeguards measurements, whether the quality of the assay will be substantially improved is questionable because the impact of DN's on the assay is predicted to be well within the overall measurement uncertainty. This is the first quantitative analysis of its kind for the impact of DN's on neutron coincidence counting.

For simplicity we restricted the discussion presented here to the familiar one-energy group model although an instrument-specific dependent three group model (fission, $(\alpha, n)$, and DN ) is strictly needed. This can be seen from the $\mathrm{S}$ equation, because, for example, the three sources require separate detection efficiencies. The value of this note is that it quantifies the influence of DN's in a simple way using the conventional point-model framework and justifies why for most routine applications of neutron coincidence counting we are justified in ignoring them. We note that the possibility exists for $\beta$-delayed double-neutron emission [21] which could contribute a delayed coincidence rate. Limited data is available to quantify this although we feel secure in neglecting it here. Our discussion follows the neutron coincidence counting tradition of known- $\alpha$, although the conclusions are more general, extension to multiplicity counting being straightforward.

\section{ACKNOWLEDGEMENT}

This work was sponsored by the U.S. Department of Energy (DOE), National Nuclear Security Administration (NNSA), Office of Nonproliferation Research and Development (NA-22). We thank Dr. Peter Santi, Los Alamos National Laboratory, and Prof. Imre Pázsit, Chalmers University of Technology, for encouragement and valuable discussions. 


\section{REFERENCES}

[1] Ensslin, N. Principles of neutron coincidence counting, Chapter 16 In: D Reilly, N Ensslin, H Smith, Jr, and S. Kreiner (Eds), Passive Nondestructive assay of nuclear materials, US Nuclear Regulatory Commission report NUREG/CR-5550 (March, 1991), ISBN 0-16-032724-5. Also known as Los Alamos National Laboratory report LA-UR-90-732.

[2] Ensslin, N., Harker, W.C., Krick M.S., Langner, D.G., Pickrell, M.M., and Stewart J.E., Application guide to neutron multiplicity counting, Los Alamos National Laboratory Report LA-UR-98-4090.

[3] Pázsit, I., Enqvist A., and Lénárd, P. A note on the multiplicity expressions in nuclear safeguards, Nucl. Instrum. and Meths. in Phys. Res A603 (2009)541-544.

[4] Bohnel, K. The Effect of Multiplication on the Quantitative determination of Spontaneously fissioining Isotopes by Neutron Correlation Analysis, Nuclear Science and Engineering 90(1985)75-82.

[5] Favalli, A., Croft, A., and Santi,P., Point model equations for neutron correlation counting: extension of Böhnel's equations to any order, Nuclear Instruments and Methods A, Vol. 795, (2015), 370-375.

[6] Cifarelli, D.M. and Hage, W., Models for a three-parameter analysis of neutron signal correlation measurements for fissile material assay. Nuclear instrument and Methods in Physics Research A251(1986)550-563.

[7] Croft,S., Favalli, A., Swinhoe, M., Goddard,B., Stewart,S., The effect of deadtime and electronics transients on the predelay bias in neutron coincidence counting, Nuclear Instruments and Methods A, Vol. 814, 2016, pages 96-103.

[8] Croft,S., Favalli,A., Dead time corrections for neutron multiplicity counting, Nuclear Instruments and Methods A, vol.686, 2012, pages 115-116.

[9] Croft,S., Favalli, A., Henzlova, D., Santi, P., On the equivalence of the RTI and SVM approaches to time correlated analysis, Nuclear Instruments and Methods A, vol.773, 2015, pages 114-116.

[10] S.Croft, A.Favalli, D.Hauck, D.Henzlova, V.Henzl, R.D. McElroy jr, P.A.Santi, Reflected-pointreactor kinematics model for neutron coincidence counting: the equation for the leakage multiplication, ESARDA bulletin no.52, (2015), pag.114-117

[11] Croft S., Favalli A., Hauck D.K., Henzlova D., Santi, P.A. Feynman variance-to-mean in the context of passive neutron coincidence counting, Nucl. Instrum. and Meths. in Phys. Res A686 (2012)136-144.

[12] Pázsit, I., On the concept of neutron multiplication, Annals of Nuclear Energy 90 (2016), 480-481 
[13] Henzlova, D., Menlove, H.O., Croft, S., Favalli, A., Santi, P., The impact of gate width setting and gate utilization factors on plutonium assay in passive correlated neutron counting, Nuclear Instruments and Methods A, Vol. 797, (2015), 144-152.

[14] Keepin G.R., Wimett T.F., Zeigler R., Delayed Neutrons from Fissionable Isotopes of Uranium, Plutonium, and Thorium, Phys.Rev.107, 1044 (1957).

[15] Walker J. and D.R. Weaver, Nuclear physics data for reactor kinetics, Advances in Nuclear Science and Technology 11(1979)1-66.

[16] Holden, N.E. and Zucker M.S. A re-evaluation of the average prompt neutron emission multiplicity (nubar), Nuclear Standard Reference Data, International Atomic Energy Agency Technical Document IAEA-TECDOC-335 (Vienna, 1985) 248-255.

[17] Zucker, M.S. and Holden, N.E., Neutron multiplicity for neutron induced fission of ${ }^{235} U,{ }^{238} U$ and ${ }^{239} \mathrm{Pu}$ as a function of neutron energy. IAEA-SM-293/122 (1987)329-347.

[18] Tuttle, R.J. Delayed-neutron yields in nuclear fission. In Proc. of the consultants' meeting on delayed neutron properties, Vienna, 26 - 30 March 1979, IAEA-INDC (NDS)-107 (Aug. 1979) 29-67.

[19] England, T.R., Brady, M.C., and Wilson, W.B. Delayed neutron spectra and intensities from evaluated precursor data, Los Alamos National Laboratory report LA-UR-85-1673, also Submitted to The International Conference on Nuclear Data for Basic and Applied Science, Santa Fe, NM, May 13-17, 1985.

[20] Pelowitz, D. et al., "MCNPX 2.7.0 Extensions", LA-UR-11-02295 (2011).

[21] Abriola, D., Singh, B., and Dillmann, I. Summary report of consultants' meeting on beta-delayed neutron emission evaluation, IAEA December2011, INDC(NDC)-0599 ; online at http://wwwnds.iaea.org/publications/indc/indc-nds-0599.pdf

[22] E. Fermi, Problem of time dependence on the reaction rate: Effect of delayed neutrons emission Report CP-291 (Notes on Lecture of October 7, 1942),Paper Number 171, pp226-229 in: Enrico Fermi, Note E Memorie (Collected Papers) Volume II, United States 1939-1954, Accademia Nazionale dei Lincei - Roma, The University of Chicago Press, (Jan., 1965). ISBN-13: 978-0226243603

[23] Uhrig, R.E., Random Noise Techniques in Nuclear Reactor Systems, The Ronald Press Company, New York, 1970.

[24] Pázsit, I, private communication, Chalmers University of Technology, Gö teborg, Sweden.

[25] Krick, M.S., Geist, W.H., and Mayo, D.R., A weighted point model for the thermal neutron multiplicity assay of high-mass plutonium samples, Los Alamos National Laboratory report, LA-14157 (Oct.2005). 